\title{
ANXIETY, DEPRESSION, AND SELF-MANAGEMENT: A SYSTEMATIC REVIEW
}

\section{Introduction and Problem}

Cardiovascular disease (CVD) consists of a group of conditions affecting the structure and function of the heart (Center for Disease Control (CDC), 2011); and if managed effectively, can lead to improved health. Coronary artery bypass grafting (CABG) and valve replacement (VR) procedures are the most common surgical treatments for cardiovascular disease (CDC). Following surgery, during the home recovery period, patients are expected to engage in the selfmanagement of behaviours to prevent complications (Robertson \& Keller, 1992). These behaviours include: managing medication and nutrition regimens, engaging in activity performance, and altering behaviours to prevent the onset and/or exacerbation of pulmonary and/or wound infections.

Despite the physical benefits associated with CABG and/or VR, approximately 29\% $61 \%$ of all patients experience moderate to high levels of anxiety and/or depression, which have been shown to remain elevated up to 6 months following surgery (Tsushima, Johnson, Lee, Matsukawa, \& Fast, 2005). Even though CABG and/or VR improves blood flow and is beneficial for patients with blocked arteries, these procedures have been linked to high rates of hospital readmissions during the first 3 months of home recovery (McCrone, Lenz, Tarzian, \& Perkins, 2001; Tsushima et al.; McKenzie, Simpson, \& Stewart, 2009). Increased readmission rates may be due to patients' inability to perform self-management behaviours which may be a result of elevated levels of post-operative anxiety and/or depression (Barnason, Zimmerman, Anderson, Mohr-Burt, \& Nieveen, 2000; Dunstan \& Riddle, 1997; Robertson \& Keller, 1992; Watt-Watson \& Stevens, 1998).

\section{Purpose of Study}


The presence of anxiety and/or depression may influence an individual's home recovery experience by hindering their ability to engage in the necessary self-management behaviours following heart surgery. Specifically, anxiety and/or depression may alter the individual's capacity to retain information, recall and use necessary resources, and to problem solve solutions to barriers encountered. To date, studies have not specifically examined the influence of anxiety and/or depression on self-management behaviour performance. Instead data pertaining to anxiety and/or depression has been collected to describe patients' psychological status following heart surgery. The objective of this review was to address the clinically relevant question: In the presence of anxiety and/or depression, do patients who have had heart surgery engage in selfmanagement behaviours following hospital discharge? Results from this study will provide a preliminary understanding of the role of anxiety and depression in influencing the performance of self-management behaviours. It will also be used to contribute to the design of future interventions for individuals diagnosed with varying degrees of anxiety and/or depression following CABG and/or VR.

\section{Conceptual Underpinnings and Literature Review}

Self-management is described as the behaviours individuals are required to engage in order to manage their conditions and/or promote recovery (Registered Nurses Association of Ontario, 2010). Specific self-management behaviours following heart surgery include: management of complications (wound, abdominal, and/or pulmonary), engagement in activity, and medication and nutritional management (McCrone, Lenz, Tarzian, \& Perkins, 2001).

Conceptually, anxiety is defined as a psychological condition "characterized by subjective, consciously perceived feelings of apprehension and tension, accompanied by activation of cognitive impulses that block judgment of various situations" (Spielberger, 1995, p. 
17). Anxiety is prevalent in patients following heart surgery and serves to obstruct the process of learning (Januzzi, Stern, Pasternak, \& DeSanctis, 2000; Mallik et al., 2005; Gortner \& Jenkins, 1990). Similar to anxiety, depression is a psychological condition that is prevalent in patients diagnosed with cardiac illnesses (Rumsfeld \& Ho, 2005). It is characterized by morbidity (i.e., hopelessness and poor quality of life), as well as increased mortality risk through pathophysiological pathways and potentially suicide.

The role of anxiety and depression in influencing outcomes after heart surgery has been explored throughout the literature (Januzzi et al., 2000; Mallik et al., 2005; Gortner \& Jenkins, 1990). Studies that have examined the association between anxiety and depression in patients following heart surgery and their risk of subsequent cardiovascular disease events (such as death, myocardial infarct, and angina) have reported inconsistent findings. Statistically significant positive relationships between post-operative anxiety and depression and myocardial infarct $(\mathrm{p}<$ $.05)$ and angina $(\mathrm{p}<.05)$ assessed at 3 months following hospital discharge have been identified (Januzzi et al.). Heightened level of anxiety at the time of discharge decreased significantly at 3 months following hospital discharge, however moderate levels still remained. However, Gortner and Jenkins, employing a randomized controlled trial, with 149 study participants found no statistically significant associations between psychological disorders (i.e. anxiety and depression) and angina, death, and myocardial infarct within 8 weeks following hospital discharge.

The findings of these studies are inconclusive yet they suggest that anxiety and depression may interfere with the home recovery process of patients who have had heart surgery. However, the influence of anxiety and/or depression on self-management behaviour performance is unknown.

\section{Methods}




\section{Study Design}

A systematic review of studies that examined the psychological condition of patients who had $\mathrm{CABG}$ and/or VR, as well as their engagement in self-management behaviours was conducted to address the study question. The research question was used to guide the specification of key terms to search databases, the identification of criteria for selecting studies, and the extraction of pertinent data. As this was a review, there were no anticipated ethical issues. The search for relevant studies was performed July 2010-January 2011 using the following databases: CINAHL, MEDLINE, PUBMED, EMBASE, COCHRANE, and HEALTH STAR. The keywords used in the search included: anxiety, depression, post-operative, selfmanagement behaviour, $\mathrm{CABG}, \mathrm{VR}$, and post-surgical. Reference lists of studies retrieved were examined for additional studies that addressed the post-operative recovery period of patients who had CABG and/or VR. Assessment of eligible studies, data extraction, and data entry were performed by the authors.

\section{Sample}

Studies were included in the review if they met the following selection criteria: 1) the sample represented adult ( $\geq 18$ years) patients who underwent CABG and/or VR surgery, 2) the outcomes assessed were related to self-management behaviours performed during the home recovery period, 3) contained data related to anxiety and/or depression, hospital readmission rates, and emergency room visits; and 4) was published in English between 2000 and 2011. Studies that used non-experimental, experimental or randomized clinical trial (RCT), and quasiexperimental designs involving two groups were included in the review.

\section{Measurement of outcome}


The following information was gathered about each study: year of publication, country in which the study was conducted, study design (non-experimental, quasi-experimental, or experimental), sample size (total, and for each study group), and demographic information pertaining to the samples identified in the studies related to age, culture, marital status, sex, education, and number and type of co-morbid conditions. Information related to the number and type of study groups (control or comparison and treatment, or two treatment groups), number and type of self-management behaviours performed, and the presence and level of severity of anxiety and depression were also extracted.

\section{Data Analysis}

Descriptive statistics were used to describe the studies included in this review in order to determine the number and type of self-management behaviours performed, and to outline the level of severity of anxiety and depression.

\section{Findings}

The 16 studies that met the inclusion criteria involved 3783 participants (Table 1). The studies were conducted in the United States (75.0\%: 12 studies) Europe (12.5\%: 2 studies), and Canada (12.5\%: 2 studies). Just over two thirds (62.5\%: 10 studies) of the studies used descriptive designs to identify self-management behaviours, anxiety, and depression, while 25.0 $\%$ (4 studies) of the studies used quasi-experimental designs, and $12.5 \%$ (2 studies) used experimental designs. Six (37.5\%) studies collected data on depression, one (6.2\%) study collected data on anxiety, and $9(56.2 \%)$ collected data on both anxiety and depression.

Across the studies, the average age of the study participants was $61.7(\mathrm{SD}=3.0)$ years. In the majority of the studies, the sample was mainly Caucasian (81.2\%: 13 studies), married (93.8 $\%: 15$ studies), male (93.8 \%: 15 studies), with at least a high school diploma (93.8 \%: 15 
studies). In $93.7 \%$ (15 studies) of the studies, the sample had less than or equal to high school education. Seventy-five percent (75.0\%: 12 studies) of the study participants were retired. Just over $80 \%$ (13 studies) of the study participants reported having at least 5 co-morbid conditions. The most frequently reported co-morbid conditions were diabetes (87.5 \%: 14 studies), hypertension (81.2\%: 13 studies), hyperlipidemia (62.5\%: 10 studies), peripheral vascular disease (50.0\%: 8 studies), and chronic obstructive pulmonary disease (43.8\%: 7 studies).

The most common complications included delirium (100\%: 16 studies), short term (last up to 2 weeks) memory loss (87.5\%: 14 studies), atrial fibrillation (56.2\%: 9 studies), heart failure (50.0\%: 8 studies), and myocardial infarct (50.0\%: 8 studies). Post-operative complications lasted between $2(\mathrm{SD}=3.8)$ to $43(\mathrm{SD}=2.9)$ days.

Thirty-eight studies were screen based on a review of the abstracts. The identified inclusion criteria were used to screen studies for inclusion in the literature review [PRISM flow diagram identifies the literature accessed, screened, and included in this review (Figure 1)].

\section{Severity of anxiety during the home recovery period}

Two studies (12.5\%) reported that participants experienced mild levels of anxiety lasting up to 6 weeks, while just over half $(62.5 \%, 10$ studies $)$ of the study samples reported moderate levels of anxiety at time of hospital discharge lasting up to one month during the home recovery period. A quarter of the samples (4 studies) reported severe anxiety at the time of hospital discharge, lasting 2 days. The level of anxiety severity gradually decreased to moderate levels of anxiety which lasted for 1 week before changing to mild anxiety continuing up to 2 weeks. The studies did not provide information as to whether or not the study participants were taking antianxiety medication. Anxiety was measured via self-report.

\section{Severity of depression during the home recovery period}


Only twelve studies (75\%) provided data pertaining to depression. Approximately two thirds (8 studies) of the studies reported mild levels of depression during the first 3 weeks following hospital discharge, while the remaining studies identified moderate levels of depression lasting up to 6 months following surgical procedure. The studies did not provide information as to whether or not the study participants were taking anti-depression medication. Depression was measured via self-report.

\section{Self-management behaviour}

On average, patients performed $4(\mathrm{SD}=0.8)$ self-management behaviours during their hospitalization and $3(\mathrm{SD}=0.8)$ self-management behaviours following hospital discharge. The three most frequently performed self-management behaviours patients engaged in during their hospitalization included deep breathing and coughing exercises (56.2\%: 9 studies), use of incentive spirometer (43.7\%: 7 studies), and activity and range of motion exercises (37.5\%: 6 studies). The three most common self-care behaviours patient engaged in after their hospital discharge were deep breathing and coughing exercises (62.5\%: 10 studies), medication administration (62.5\%: 10 studies), and medication management in particular refilling prescriptions on time (50.0\%: 8 studies). Individuals with mild levels of anxiety and depression engaged in a variety of self-management behaviours ( $n=3$ studies $)$, over a period of time including management of complications, medication administration, activity and range of motion exercise, and nutrition management. However, individuals with moderate to severe levels of anxiety were only able to engage in one self-management behaviour at a specific point in time. Levels of depression in these individuals were minimal. Of note, there is no evidence to suggest that this behaviour was repeated over time. Changes in degree of severity of anxiety and depression from moderate and severe to mild was associated with an increase in the number of 
self-management behaviours $(\mathrm{n}=2$ studies) performed. There were no consistent themes pertaining to type of self-management behaviours performed based on level of severity of anxiety and depression.

\section{Discussion and Application}

In the 16 studies of this review, at least half reported moderate to severe levels of anxiety and depression were reported during the home recovery period. Moderate anxiety and depression scores are consistent with expectations and with previous findings in similar (McCrone, Lenz, Tarzian, \& Perkins, 2001) and different patient populations (Reuber, Andersen, Leger, \& Helmstaedter, 2004; Korfage, Essink-Bot, Janssens, Schro“der, \& de Koning, 2006) that included individuals diagnosed with epilepsy and prostate cancer.

Individuals with mild anxiety and/or depression engaged in a higher number of selfmanagement behaviours than individuals with moderate to severe anxiety and depression. When anxiety and depression levels changed from moderate and severe to mild, the number of selfmanagement behaviours engaged in increased. This may be explained by the theoretical supposition that the performance of self-management behaviours is influenced by an individual's ability to acquire, retain, recall, and implement knowledge, which in turn is influenced by psychological factors such as anxiety and depression (Orem, 2001). The more intense the presence of anxiety and depression, the less likely an individual will be able to engage in activity.

Moderate to severe levels of anxiety and/or depression exist during the first month of recovery from CV surgery and can last up to 6 months. Anxiety and depression influences the number of self-management behaviours patients will engage in to facilitate their home recovery process. This is problematic as the first month of home recovery is considered to be the time 
when patients are most prone to the development of complications (i.e. fluid overload, irregular heart beat, and infections) and re-hospitalizations (XXX, 2008). As investigators are continuing to examine the influence of anxiety and/or depression on patient outcomes following heart surgery, it is important for clinicians to incorporate the management of these psychological conditions into their overall plan of care. Hence, clinicians should consider assessing patients' anxiety and depression levels immediately following their hospital discharge and during their routine follow-up visits to the cardiologist and surgeon. Individuals identified as having moderate to severe levels of anxiety and/or depression should be followed up on a routine basis (i.e. at least once weekly) during the first month of home recovery to continually monitor their psychological status as it may change over the course of the individual's home recovery. Based on the findings of this review, it appears that moderate to severe levels of anxiety and depression may impede an individual's ability to engage in self-management behaviours. Thus, it may be unreasonable for nurses to expect patients with moderate to severe levels of anxiety and depression to participate in self-management behaviours during their initial home recovery period without attention to the anxiety and depression. Based on the results, 10 of the studies reviewed indicated patients had moderate levels of anxiety, while 4 studies identified patients with moderate to severe depression during the first month of recovery. Thus, instead of implementing interventions that address self-management behaviour performance during the home recovery period, clinicians should consider implementing interventions aimed at decreasing the level of severity of both anxiety and depression first, and then introduce selfmanagement behaviour interventions gradually as anxiety and depression resolve.

Spielberger's State-Trait Anxiety Tool (1995) and Beck Depression Inventory (Beck, Rial, Rickels, 1974) are two assessment tools that nurses can use to assess anxiety and 
depression in individuals recovery from heart surgery. Both instruments have been evaluated and shown to be reliable and valid in CVS populations (Beck, Rial, Rickel; Spielberger).

\section{Conclusion and Implications for Future Research}

For most home post-operative patients who have had CVS, moderate to severe levels of anxiety and depression exist during the first month of home recovery and appear to have an effect on the performance of self-management behaviours. Music and relaxation therapy as evidenced-based recommendations may reduce anxiety and depression in patients following heart surgery. The results reported are based on a review. Continued examination of postoperative anxiety and depression during the home recovery period is required to replicate findings from the review. A descriptive, longitudinal study should be conducted to determine the severity and duration of anxiety and depression during the home recovery period. Once a better science bases exists linking anxiety and depression to self-management behaviours, then focused evidenced-based interventions (including music and relaxation techniques) can be developed to meet the needs of home care patients following CVS. As well, once findings have been replicated, integration of the two evidence based interventions into clinical practice is suggested. 


\section{References}

Ai, A., Dunkle, R., Peterson, C., Saunders, D., \& Bolling, S. (1998). Self-care and psychosocial adjustment of patients following cardiac surgery. Social Work Health Care, 27, 75-95.

Barnason, S., Zimmerman, L., Anderson, A., Mohr-Burt, S., \& Nieveen, J. (2000). Functional status outcomes of patients with a coronary artery bypass graft over time. Heart and Lung, 29, 33-46.

Barnason, S., Zimmerman, L., \& Nieveen, J. (1995). The effects of music interventions on anxiety in the patient after coronary artery bypass grafting. Heart and Lung, 24(2), 124132.

Beck, A. T., Rial, W. Y., \& Rickels, K. (1974). Short form of depression inventory: Cross validation. Psychological Reports, 34, 1184-1 186.

Blumenthal, J., Lett, H., Babyak, M., White, W., Smith, P., Mark, D., Jones, R., Mathew, J. \& Newman, M. (2003). Depression as a risk factor for mortality after coronary artery bypass surgery. Lancet, 362, 604-609.

Borowicz, L., Royall, R., Grega, M., Selnes, O., Lyketsos, C., \& McKhann, G. (2002). Depression and cardiac morbidity 5 years after coronary artery bypass surgery. Psychosomatics, 43, 464-471.

Burg, M. M., Benedetto, M. C., Rosenberg, R., \& Soufer, R. (2003). Presurgical depression predicts medical morbidity 6 months after coronary artery bypass graft surgery. Psychosomatic Medicine, 65, 111-118.

Burg, M. M., Benedetto, M. C., \& Soufer, R. (2003). Depressive symptoms and mortality two years after coronary artery bypass graft surgery (CABG) in men. Psychosomatic Medicine, 65, 508-510. 
Center for Disease Control. Heart disease. Coronary artery disease. Available at: http://www.cdc.gov/heartdisease/coronary_ad.htm (accessed October 18, 2011).

Dunstan, J. L., \& Riddle, M. M. (1997). Rapid recovery management: The effects on the patient who has undergone heart surgery. Heart and Lung, 26, 289-298.

Gortner, S. \& Jenkins, C. D. (1990). Self-efficacy and activity level following cardiac surgery. Journal of Advanced Nursing, 15, 1132-1138.

Hanser, S. B., \& Thompson, L. W. (1994). Effects of a music therapy strategy on depressed older adults. Journal of Gerontology, 49(6), 265-269.

Januzzi, J. L., Stern, T. A., Pasternak, R. C., \& DeSanctis, R. W. (2000). The influence of anxiety and depression on outcomes of patients with coronary artery disease. Archives of Internal Medicine, 160, 1913-1921.

Jenkins, C. D., Stanton, B. A., Savageau, J. A., Denlinger, P., \& Klein, M. D. (1983). Coronary artery bypass surgery. Physical, psychological, social, and economic outcomes six months later. Journal of the American Medical Association, 250, 782-788.

Korfage, I. J., Essink-Bot, M-L., Janssens, A., Schro“der, F. W., \& de Koning, H. J. (2006). Anxiety and depression after prostate cancer diagnosis and treatment: 5-year follow-up. British Journal of Cancer, 94, 1093-1098.

Mallik, S., Krumholz, H. M., Lin, Z. Q., Kasl, S. V., Mattera, J. A., Roumains, S. A., \& Vaccarino, V. (2005). Patients with depressive symptoms have lower health status benefits after coronary artery bypass surgery. Circulation, 111, 271-277.

McCrone, S., Lenz, E., Tarzian, A., \& Perkins, S. (2001). Anxiety and depression: Incidence and patterns in patients after coronary artery bypass graft surgery Applied Nursing Research, $14,155-164$. 
McGrady, A., McGinnis, R., Badenhop, D., Bentle, M., \& Rajput, M. (2009). Effects of depression and anxiety on adherence to cardiac rehabilitation. Journal of Cardiopulmonary Rehabilitation, 29, 358-364.

McKenzie, L. H., Simpson, J., \& Steward, M. (2009). The impact of depression on activities of daily living skills in individuals who have undergone coronary artery bypass graft surgery. Psychology, Health \& Medicine, 14(6), 641-653.

McKenzie, L. H., Simpson, J., \& Stewart, M. (2010). A systematic review of pre-operative predictors of post-operative depression and anxiety in individuals who have undergone coronary artery bypass graft surgery. Psychological Health Medicine, 15, 74-93.

Orem, D. E. (2001). Nursing: Concepts of Practice. $5^{\text {th }}$ ed. St. Louis: Mosby.

Rafanelli, C., Roncuzzi, R., \& Milaneschi, Y. (2006). Minor depression as a cardiac risk factor after coronary artery bypass surgery. Psychosomatics, 47, 289-295.

RNAO (September 2010). Strategies to support self-management in chronic conditions: Collaboration with clients. RNAO: Toronto, Canada.

Reuber, M., Andersen, B., Leger, C. E., \& Helmstaedter, C. (2004). Depression and anxiety before and after temporal lobe epilepsy surgery. Seizure, 13, 129-135.

Robertson, D., \& Keller, C. (1992). Relationships among health beliefs, self-efficacy, and exercise adherence in patients with coronary artery disease. Heart and Lung, 21, 56-63.

Rumsfeld, J.S. \& Ho, PM. (2005). Depression and cardiovascular disease. Circulation, 111, 250253.

Spielberger, C. D. (1995). Test Anxiety: Theory, Assessment, and Treatment. Washington, D. C.: Taylor \& Francis. 
Tsushima, W. T., Johnson, D. B., Lee, J. D., Matsukawa, J. M., Fast, K. M. S. (2005). Depression, anxiety, and neuropsychological test scores of candidates for coronary artery bypass graft surgery. Archives of Clinical Neuropsychology, 20(5), 667-673.

Tully, P. J., Baker, R. A., Turnbull, D. A., Winefield, H. R., \& Knight, J. L. (2009). Negative emotions and quality of life six months after cardiac surgery: The dominant role of depression not anxiety symptoms. Journal of Behavioral Medicine, 32, 510-522.

Tully, P. J. , Pederson, S. S., Winefield, H. R., Baker, R. A., Turnbull, D. A., \& Denollet, J. (2011). Cardiac morbidity risk and depression and anxiety: A disorder, symptom and trait analysis among cardiac surgery patients. Psychology, Health \& Medicine, 16(3) 333345.

Van Dixhoorna, J. \& White, A. (2005). Relaxation therapy for rehabilitation and prevention in ischaemic heart disease: a systematic review and meta-analysis. Journal of Cardiovascular Risk, 12, 193-202.

Vingerhoets, G. (1998) Perioperative anxiety and depression in open-heart surgery. The Academy of Psychosomatic Medicine, 39, 30-37.

Watt-Watson, J., \& Stevens, B. (1998). Managing pain after Coronary Artery Bypass surgery. Journal of Cardiovascular Nursing, 12, 39-51.

This manuscript was accepted for publication by Sage Publication, Fredericks, S., Lapum, J., Lo, J. (2012). Anxiety, depression, and self-management: A systematic review. Clinical Nursing Research: An International Journal, 21(4), 411-429. doi 10.1177/1054773812436681. 